\title{
A Note on the Selection Expectation and Support Function
}

\author{
Rigao He \\ Department of Mathematics, College of Science, Hunan Institute of Engineering, Xiangtan, China \\ Email: rg@shu.edu.cn
}

Received 12 July 2015; accepted 9 August 2015; published 12 August 2015

Copyright (C) 2015 by author and Scientific Research Publishing Inc.

This work is licensed under the Creative Commons Attribution International License (CC BY).

http://creativecommons.org/licenses/by/4.0/

cc) (i)

Open Access

\begin{abstract}
In this paper, we prove the relationship between selection expectation and support function by a new method.
\end{abstract}

Keywords

Support Function, Hausdorff Metric, Random Set, Selection Expectation

\section{Introduction}

The studies of random geometrical objects can go back at least to the famous Buffon needle problem [1]. Then the theory of random sets first study in the book by Matheron [2], who formulated the exact definition of a random closed set and developed the relevant techniques. The recently published book by Molchanov [1] is highly interdisciplinary and unites a number of mathematical theories and concepts for stochastic geometry, which has witnessed a rapid growth (see, e.g., [3]-[12]).

The relationship between random sets and convex geometry has been thoroughly explored within the stochastic geometry literature; see, e.g. Weil and Wieacker [13]. The main techniques stem from convex and integral geometry; see Schneider [14], Gardner [15] and Schneider and Weil [8]. The support function is one of the most important concepts in convex geometry. The goal of the present paper is to discuss a new approach for the relationship between selection expectation and support function, which has played an essential role in proving the strong law of large numbers for random compact sets [4].

The organization of this manuscript is as follows. In the next section, we set notations and give preliminaries. In the last section we will prove the relationship between selection expectation and support function by a new method. 


\section{Notations and Preliminaries}

We consider the $d$-dimensional Euclidean space $\mathbb{R}^{d}$ equipped with its usual inner product $\langle$, $\rangle$, norm $\|\cdot\|$, the unit sphere $S^{d-1}$ and the unit ball $B$.

Let $\mathcal{K}$ denote the family of all nonempty, compact subsets of $\mathbb{R}^{d}, \mathcal{K}_{c}$ denote the subfamily of $\mathcal{K}$ which are also convex.

For $K \in \mathcal{K}, u \in S^{d-1}$, the support function [14] [15] of $K$ is defined by

$$
h(K, u)=\max \{\langle x, u\rangle \mid x \in K\} .
$$

Obviously, for $K, \quad L \in \mathcal{K}_{c}$,

$$
K \subseteq L \text { if and only if } h(K, u) \leq h(L, u) .
$$

Hence a convex body is uniquely determined by its support function.

In order to show that a sequence of the sets of nonempty, compact subsets converges to another set of nonempty, compact subsets. One must define the distance between two sets. It motivates the following definition.

For $K, L \in \mathcal{K}$, Hausdorff metric (Hausdorff distance) [14] [15] between $K$ and $L$ is defined as

$$
\rho(K, L)=\min \{\lambda \geq 0 \mid K \subseteq L+\lambda B, L \subseteq K+\lambda B\}=\sup _{u \in S^{n-1}}|h(K, u)-h(L, u)| .
$$

Then $\mathcal{K}$ turns into a separable, locally compact metric space.

The point $x \in \mathbb{R}^{d}$ is a convex combination [14] [15] of the points $x_{1}, \cdots, x_{m} \in \mathbb{R}^{d}$, if there are numbers $\lambda_{1}, \cdots, \lambda_{m} \in \mathbb{R}$ such that

$$
x=\lambda_{1} x_{1}+\cdots+\lambda_{m} x_{m}, \lambda_{i} \geq 0(i=1, \cdots, m), \sum_{i=1}^{m} \lambda_{i}=1 .
$$

The set of all convex combinations of any finitely many elements of $A$ is called the convex hull of $A$ and is denoted by $\operatorname{co} A$.

The family of closed subsets of $\mathbb{R}^{d}$ is denoted by $\mathcal{F}$. Let us fix a complete probability space $(\Omega, \mathfrak{F}, P)$ which will be used to define random elements ([1] [16]).

A map $X: \Omega \rightarrow \mathcal{F}$ is called a random closed set if, for $K \in \mathcal{K}$,

$$
\{\omega: X(\omega) \cap K \neq \varnothing\} \in \mathfrak{F} .
$$

It is natural to define random open sets as complements to random closed sets, so that $Y: \Omega \rightarrow \mathcal{F}$ is called a random open set if its complement $X=Y^{c}$ is a random closed set.

Therefore we can regard a random set $X$ as a measurable map defined on an abstract probability space $(\Omega, \mathfrak{F}, P)$ and taking values in the collection $\mathcal{K}$.

A random set $X$ is called simple, if there exists a finite measurable partition $A_{1}, A_{2}, \cdots, A_{n}$ of $\Omega$ and sets $F_{1}, F_{2}, \cdots, F_{n} \in \mathcal{F}$ such that $X(\omega)=F_{i}$ for all $\omega \in A_{i}, 1 \leq i \leq n$.

A random set $X$ is called approximable if $X$ is an almost sure limit of a sequence of simple random sets.

Similarly, a random set $X$ with almost surely compact values is called a random compact set.

The norm $\|X\|$ of a random set $X$ is the real random variable associating the distance from the origin to every $X(\omega)$, i.e.

$$
\|X\|=\sup \{\|x\|, x \in X\} \text {. }
$$

A random vector $x: \Omega \rightarrow \mathcal{K}$ is a selection of $X$ if $P(x \in X)=1$.

$A \in \mathfrak{F}$ is an atom in probability space $(\Omega, \mathfrak{F}, P)$, if $P(A)>0$ and for any $A^{\prime} \in \mathfrak{F}$ with $A^{\prime} \subseteq A$, either $P\left(A^{\prime}\right)=P(A)$ or $P\left(A^{\prime}\right)=0$. The probability space is said to be non-atomic if no such $A$ exists.

The space $\mathcal{F}$ of closed sets (and also the space $\mathcal{K}$ of compact sets) is non-linear, so that conventional concepts of expectations in linear spaces are not directly applicable for random closed (or compact) sets.

Following Artstein and Vitale [4] in adapting the Aumann [16] integral, the expectation of $X$ is defined by

$$
E X=\{E x \mid x \text { is a selection of } X \text { and } E\|x\|<\infty\} \text {. }
$$

Remark: 1) Selection expectation (also called the Aumann expectation), which is the best investigated con- 
cept of expectation for random sets. Since many results can be naturally formulated for random closed sets in Banach spaces.

2) We cite an example to illustrate the meaning of selection expectation. If $X$ is a simple random compact set, i.e. $X=K_{1} \mathcal{X}_{A_{1}}+\cdots+K_{m} \mathcal{X}_{A_{m}}$, where $K_{i} \in \mathcal{K}, A_{i} \in \mathfrak{F}$ and $\mathcal{X}_{A_{i}}$ is the characteristic function of $A_{i}$, then

$$
E X=P\left(A_{1}\right) K_{1}+\cdots+P\left(A_{m}\right) K_{m} .
$$

3) $E X \in \mathcal{K}$ is equivalent to $E\|X\|<\infty$.

4) Moreover, if the probability space $(\Omega, \mathfrak{F}, P)$ is nonatomic, then $E X=E \operatorname{co} X$ (see, for instance, Artstein [3] and Aumann [16]).

\section{Selection Expectation and Support Functions}

Now we are ready to prove the main result in this section.

Theorem ([1], p. 159, Theorem 1.26). If $(\Omega, \mathfrak{F}, P)$ is nonatomic, then the selection expectation of $X$ is the unique convex closed set EX satisfying

$$
E h(X, u)=h(E X, u), \text { for all } u \in S^{n-1} .
$$

Proof. One of two probability spaces contains the atoms which may lead to the fact that two independent and identically distributed random compact sets may have different selection expectations. Therefore if $X_{i}$ is nonconstant, we can pick appropriate probability space such that the probability space is nonatomic.

Note that if $X$ is a random compact set, then $h(X, u)$ is random variable.

For $X: \Omega \rightarrow \mathcal{K}$, there exists a sequence of simple random compact sets $Y_{n} \rightarrow X$. Then we can infer that

$$
E Y_{n} \rightarrow E X .
$$

Let $Y_{n}=K_{1}^{n} \mathcal{X}_{A_{1}^{n}}+\cdots+K_{m_{n}}^{n} \mathcal{X}_{A_{m_{n}}^{n}}$, where $K_{i}^{n} \in \mathcal{K}, A_{i}^{n} \in \mathfrak{F}$ and $\mathcal{X}_{A_{i}^{n}}$ is the characteristic function of $A_{i}^{n}\left(1 \leq i \leq m_{n}\right)$. From the definition of Hausdorff metric, the continuity and the linearity of the support function and (5), it follows that

$$
\begin{aligned}
E h(X, u) & \leftarrow E h\left(Y_{n}, u\right) \\
& =E h\left(K_{1}^{n} \mathcal{X}_{A_{1}^{n}}+\cdots+K_{m_{n}}^{n} \mathcal{X}_{A_{m_{n}}^{n}}, u\right) \\
& =E\left(h\left(K_{1}^{n} \mathcal{X}_{A_{1}^{n}}, u\right)+\cdots+h\left(K_{m_{n}}^{n} \mathcal{X}_{A_{m_{n}}^{n}}, u\right)\right) \\
& =E h\left(K_{1}^{n} \mathcal{X}_{A_{1}^{n}}, u\right)+\cdots+E h\left(K_{m_{n}}^{n} \mathcal{X}_{A_{m_{n}}^{n}}, u\right) \\
& =P\left(A_{1}^{n}\right) h\left(K_{1}^{n}, u\right)+\cdots+P\left(A_{m_{n}}^{n}\right) h\left(K_{m_{n}}^{n}, u\right) \\
& =h\left(P\left(A_{1}^{n}\right) K_{1}^{n}, u\right)+\cdots+h\left(P\left(A_{m_{n}}^{n}\right) K_{m_{n}}^{n}, u\right) \\
& =h\left(P\left(A_{1}^{n}\right) K_{1}^{n}+\cdots+P\left(A_{m_{n}}^{n}\right) K_{m_{n}}^{n}, u\right) \\
& =h\left(E Y_{n}, u\right) \\
& \rightarrow h(E X, u) .
\end{aligned}
$$

It follows that

$$
E h(X, u)=h(E X, u), \quad \text { for all } u \in S^{n-1} .
$$

\section{Acknowledgements}

The authors would like to acknowledge the support from the Educational Commission of Hunan Province of China (12A033) and the Hunan Provinial Natural Science Foundation of China (14JJ2122). 


\section{References}

[1] Molchanov, I.S. (2005) Theory of Random Sets. Springer, London.

[2] Matheron, G. (1975) Random Sets and Integral Geometry. Wiley, New York.

[3] Artstein, Z. (1974) On the Calculus of Set-Valued Functions. Indiana University Mathematics Journal, 24, $433-441$. http://dx.doi.org/10.1512/iumj.1975.24.24034

[4] Artstein, Z. and Vitale, R.A. (1975) A Strong Law of Large Numbers for Random Compact Sets. Annals of Probability, 5, 879-882. http://dx.doi.org/10.1214/aop/1176996275

[5] Cressie, N. (1978) A Strong Limit Theorem for Random Sets. Advances in Applied Probability, 10, 36-46. http://dx.doi.org/10.2307/1427005

[6] Kendall, D.G. (1974) Foundations of a Theory of Random Sets. In: Harding, E.F. and Kendall, D.G., Eds., Stochastic Geometry, Wiley, New York.

[7] Puri, M.L. and Ralescu, D.A. (1983) Strong Law of Large Numbers for Banach Space Valued Random Sets. Annals of Probability, 1, 222-224. http://dx.doi.org/10.1214/aop/1176993671

[8] Schneider, R. and Weil, W. (2000) Stochastische Geometrie. Teubner, Leipzig. http://dx.doi.org/10.1007/978-3-322-80106-7

[9] Vitale, R.A. (1990) The Brunn-Minkowski Inequality for Random Sets. Journal of Multivariate Analysis, 33, $286-293$. http://dx.doi.org/10.1016/0047-259X(90)90052-J

[10] Vitale, R.A. (1991) Expected Absolute Random Determinants and Zonoids. Annals of Applied Probability, 1, $293-300$. http://dx.doi.org/10.1214/aoap/1177005938

[11] Vitale, R.A. (1995) On the Volume of Parallel Bodies: A Probabilistic Derivation of the Steiner Formula. Advances in Applied Probability, 27, 97-101. http://dx.doi.org/10.2307/1428098

[12] Vitale, R.A. (1996) The Wills Functional and Gaussian Processes. Annals of Probability, 24, 2172-2178. http://dx.doi.org/10.1214/aop/1041903224

[13] Weil, W. and Wteacker, J.A. (1993) Stochastic Geometry. In: Gruber, P.M. and Wills, J.M., Eds., Handbook of Convex Geometry, Elsevier Sci. Publ., North-Holland, 1393-1438. http://dx.doi.org/10.1016/b978-0-444-89597-4.50023-8

[14] Schneider, R. (1993) Convex Bodies: The Brunn-Minkowski Theory. Cambridge University Press, Cambridge. http://dx.doi.org/10.1017/CBO9780511526282

[15] Gardner, R.J. (1995) Geometric Tomography. Cambridge University Press, Cambridge.

[16] Aumann, R.J. (1965) Integrals of Set-Valued Functions. Journal of Mathematical Analysis and Applications, 12, 1-12. http://dx.doi.org/10.1016/0022-247X(65)90049-1 